\title{
Un modelo de formación y desarrollo profesional docente para una educación científica de calidad para jóvenes en situación de vulnerabilidad social
}

\author{
Elsa Meinardi* \\ Artículo recibido: 29-04-2009 y aprobado: 12-11-2009
}

\begin{abstract}
A formation model and educational professional development for a scientific education of quality of young in situation of social vulnerability
\end{abstract}

Resumen: La inequidad social se plantea como la principal barrera para el acceso a la escolarización. También la propia escuela puede transformarse en expulsiva, sin capacidad de retención de los y las jóvenes de los sectores más desfavorecidos, de aquellos que son socialmente más vulnerables. En muchos casos puede deberse a que los docentes no poseen herramientas adecuadas para la educación en tales contextos, ya que la formación profesional los prepara muy pocas veces para ello.

De allí que el objetivo general de nuestro trabajo es contribuir a la formación de docentes que cuenten con un conocimiento de los contextos educativos, sociales, individuales e institucionales de escuelas que atienden a jóvenes en situación de vulnerabilidad social extrema, con el fin de desarrollar un profesional reflexivo, capaz de encarar de manera creativa una educación científica de calidad, que al mismo tiempo mejore la retención de los jóvenes.

Palabras clave: Formación docente para la equidad, inclusión educativa con calidad.
Abstract: Social inequality is the main barrier to access to schooling. Also the school itself can become an expulsive, without holding capacity of young people from disadvantaged sectors, those most socially vulnerable. In many cases it may be because teachers do not have adequate tools for education in such contexts because seldom training prepares them for that.

The overall objective of our work is to help train teachers who have a knowledge of the educational, social, institutional and individual schools that serve young people in extreme situation of social vulnerability, in order to develop a reflective professional able to deal creatively a quality science education, while improving the retention of young people.

Key words: Teacher training for equity, inclusive education with quality.

\footnotetext{
Centro de Formación e Investigación en Enseñanza de las Ciencias. Facultad de Ciencias Exactas y Naturales, Universidad de Buenos Aires, Argentina.emeinardi@fibertel.com.ar
} 


\section{Romper el círculo de la exclusión}

La desigualdad de oportunidades en la educación es un factor determinante en el acrecentamiento de la pobreza, el hambre y la mortalidad infantil en todo el mundo. Según datos recientes de la UNESCO (2008), el fracaso de los gobiernos en la lucha contra las profundas y persistentes desigualdades educativas, condena a millones de niños a vivir en la pobreza en el futuro. La falta de recursos, el aislamiento geográfico, el sexo, el idioma y la etnia son los principales obstáculos en el camino hacia la escolarización, destacándose como denominador común, la desigualdad de oportunidades.

El documento sobre el Seguimiento de la Educación en el Mundo, publicado este año (AA.VV., 2009) -titulado "Superar la desigualdad: por qué es importante la gobernanza"- advierte que una serie de disparidades "inaceptables" tanto en el ámbito nacional como internacional, socavan los esfuerzos a favor del desarrollo. El informe habla del profundo abismo que separa los países pobres de los ricos en lo que respecta a la igualdad de oportunidades educativas y advierte que las estadísticas relativas a los niños sin escolarizar sólo son un indicador parcial de la magnitud del problema.

¿Por qué los datos respecto de los niños y niñas sin escolarizar muestran sólo una parte del problema? Porque no tienen en cuenta los que ingresan en la escuela y desertan prematuramente, sin terminar sus estudios, y tampoco considera que las evaluaciones del rendimiento escolar de los alumnos que permanecen en la escuela dan pruebas sólidas del fracaso de los sistemas es- colares en el logro de una educación de buena calidad.

Podemos sintetizar diciendo que la educación se enfrenta al problema de que millones de niños y niñas que no ingresan al sistema, e incluso en el caso de los niños de los sectores más vulnerables socialmente que se encuentran institucionalizados, no tienen asegurada su retención y la calidad de sus aprendizajes. En Argentina, para citar la situación que más conozco, viven aproximadamente 11 millones de jóvenes, de los cuales el $40 \%$ se halla en situación de pobreza, más del 40\% está en situación de exclusión o vulnerabilidad educativa y el $20 \%$ se caracteriza como excluido del sistema educativo (INDEC, censo 2001). La Ciudad de Buenos Aires no es ajena a esta situación: casi el 30\% de los jóvenes se hallan en situación de vulnerabilidad educativa y poco menos del 10\% en exclusión educativa (datos de la Fundación SES, 2007).

Analizando las tasas netas de escolarización secundaria en Argentina (Tabla 1), es decir, el número de jóvenes efectivamente escolarizados, sobre el total de jóvenes en edad escolar, es posible identificar claramente el mapa de pobreza del país, ya que las tasas más bajas de escolarización se registran en las cinco provincias más pobres (INDEC, 2001). Como se deduce del gráfico, lamentablemente la situación general para América Latina y el Caribe, no es más alentadora y en algunos países es francamente grave. 


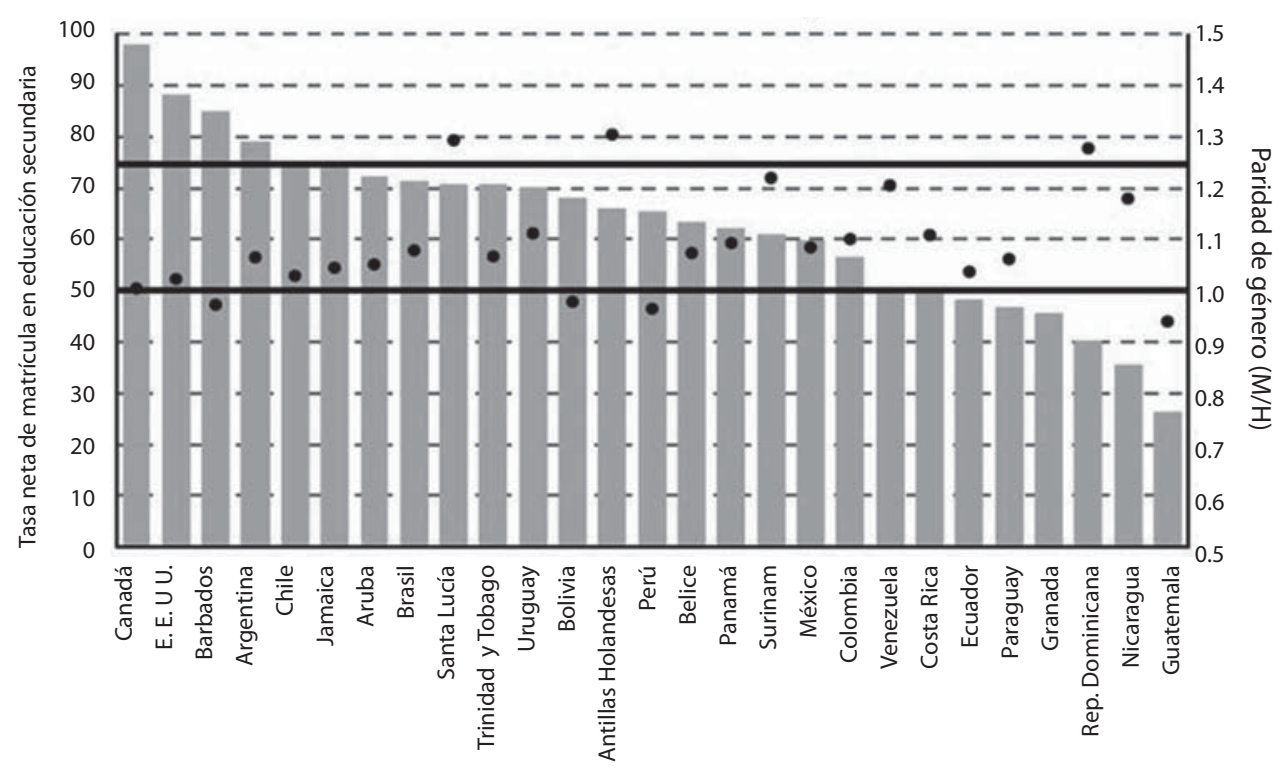

Tabla 1. Alcanzando las Metas Educativas. Fuente: OREALC, 2003 Tasa neta de matrícula en educación secundaria y paridad de género [año académico iniciado en el 2000]
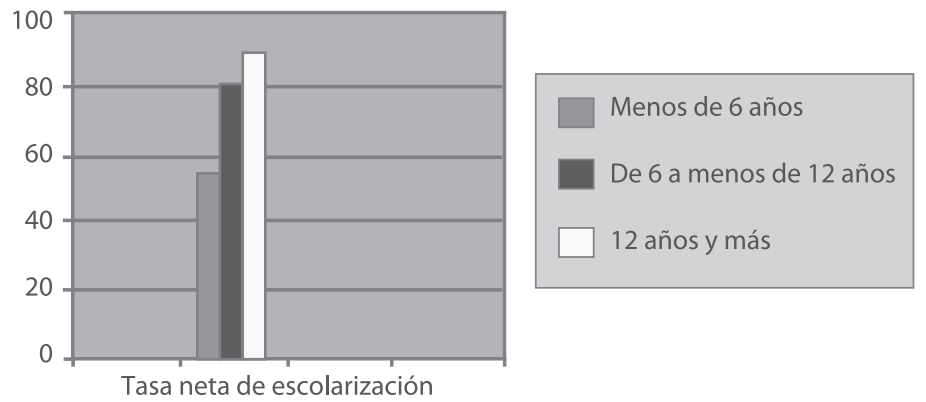

Tabla 2. Tasa neta de escolarización por clima educativo del hogar. Datos para Argentina, 2003. Fuente: SITEAL, 2008.

Sumado a esto, se ha visto, y esto refiere a datos de toda la región (América Latina y el Caribe), que los años de escolarización están estrechamente ligados al clima educativo del hogar.

Menos del 50\% de los niños y niñas se encuentran escolarizados en aquellas familias cuyos miembros no han completado los estudios de nivel primario. Cuando la escolarización familiar alcanza la finalización de estudios de nivel primario y hasta algunos años de escuela media, el valor asciende al $80 \%$, mientras que en familias con algún año de estudios terciarios el 90\% de los jóvenes está escolarizado.

Estas cifras no hacen más que mostrarnos la necesidad de retener, con calidad, a los y las estudiantes, ya que cuando la escuela se torna expulsiva está excluyen- 
do preferentemente a los niños y niñas de las familias más desfavorecidas. Según Duro (2005):

Las demandas para la política pública en el sector educativo en la región abarcan: la necesaria mejora de la calidad para lograr que los que están en el sistema permanezcan y alcancen a egresar con resultados significativos y pertinentes; atender específicamente a la población que está en situación de riesgo y buscar estrategias innovadoras, sin excluir la complejidad inherente a los desafíos de calidad y equidad.

\section{Múltiples formas de exclusión}

La exclusión de la escuela, esto es, la no escolarización de jóvenes que tienen estudios medios incompletos, es una de las formas más contundentes del problema de la exclusión educativa. Sin embargo, es importante tener presente que la fragmentación del sistema educativo genera situaciones de inclusión de baja calidad, o calidad poco significativa, en la escuela para los jóvenes de algunos grupos sociales. Esto nos lleva a concluir que estamos ante un fenómeno de inclusión en el sistema educativo, pero de exclusión de la educación con calidad (Borzese y Bottinelli, 2005). Incluir implica, además de aumentar la participación, brindar una educación de calidad (Jacinto y Terigi, 2007).

Gentili habla de un "proceso de escolarización marcado por una dinámica de exclusión incluyente"; esto es, un proceso mediante el cual los mecanismos de exclusión educativa se recrean y asumen nuevas fisonomías, en el marco de dinámicas de inclusión o inserción institucional que acaban resultando insuficientes o, en algunos casos, inocuas para revertir los procesos de aislamiento, marginación y negación de derechos que están involucrados en todo proceso de segregación social, dentro y fuera de las instituciones educativas (Gentili, 1998 y 2007; Gentili y Alencar, 2001).

\section{¿Quiénes son los niños excluidos de la escuela?} Los que cuentan con historias de repitencia, los migrantes, los que se reinsertan, las adolescentes embarazadas, los que presentan sobreedad, los niños con causas judiciales y en situación de libertad asistida, los pertenecientes a comunidades indígenas, los pobres, los que viven en situación de calle, los "malos alumnos", los alumnos difíciles, los que "se portan mal"... es decir, los niños que asisten a las escuelas para las que formamos profesores.

\section{¿También son niños a los que la escuela excluye?}

Gentili (2009) vuelve a ser muy claro al respecto cuando menciona que los que están excluidos del derecho a la educación, no lo están solo por permanecer fuera de la escuela, sino también por ser parte de un conjunto de relaciones y circunstancias que los alejan de este derecho, negándoselo o atribuyéndoselo de forma restricta, condicionada o subordinada. Históricamente, a los pobres se les ha negado el derecho a la educación impidiéndoles el acceso a la escuela.

Hoy se les niega este derecho al no ofrecerles otra alternativa sino la de permanecer en un sistema educativo que no garantiza ni crea condiciones para el acceso efectivo a una educación de calidad, al limitar las condiciones efectivas de ejercicio de este derecho por la persistencia de las condiciones 
de exclusión y desigualdad que se han transferido hacia el interior del mismo sistema escolar. Estas condiciones bloquean, obstaculizan y limitan la eficacia democrática del proceso de expansión educativa que además condujo a los pobres hacia el interior de una institución que, en el pasado cercano, disponía de un conjunto de barreras que limitaban sus oportunidades de acceso y permanencia.

Todos tienen el mismo derecho a la educación, pero no todos tienen el mismo derecho a la escuela, por lo que los resultados del proceso de escolarización son tan desiguales como son desiguales las condiciones de vida de las personas (Gentili, 2009). Según el autor, las condiciones observacionales de este proceso son bien conocidas y estudiadas en América Latina, como por ejemplo:

- Diferenciación en el acceso educativo.

- Discriminación pedagógica y logros escolares desiguales en virtud de criterios de género, desigualdades regionales, raciales o étnicas.

- Heredad de las oportunidades educativas.

- Desigualdad en la calidad educativa.

- Injusticia curricular.

- Desigualdad en la distribución de las oportunidades educativas: docentes diferentes, escuelas diferentes, recursos pedagógicos diferentes, competencias diferentes, aprendizajes diferentes, expectativas educativas diferentes; éxitos y fracasos diferentes.

\section{Cuando la formación docente es un factor más de expulsión}

Sumado a las dificultades de acceso a la escolarización que mencionamos, resulta cada vez más evidente que la propia escuela pueda transformarse en una institución expulsiva, sin capacidad de retención de los y las jóvenes de los sectores más desfavorecidos, de aquellos más vulnerables socialmente. En muchos casos puede deberse a que los docentes no poseen herramientas adecuadas para la educación en tales contextos ya que pocas veces la formación profesional los prepara para ello.

Sucede así que los profesores y profesoras dejan rápidamente estas instituciones -también es alto el ausentismo y la rotación docente (Jacinto y Terigi, 2007)- produciéndose situaciones que muchas veces los jóvenes viven como abandono y que parecen repetir aquellas que sufren en su entorno familiar y social (Meinardi, 2007). Croce (2005) señala que en numerosas oportunidades han hallado docentes que dicen con preocupación o resignación: "no hemos sido preparados ni formados para trabajar con estos jóvenes o en estos ambientes".

Feldman (2005) menciona que el modelo pedagógico vigente en las escuelas medias es incapaz de modificar los actuales problemas de inclusión educativa. También menciona que las modalidades de trabajo más extendidas en las escuelas no parecen aptas para atenuar los problemas de fracaso escolar. Según Krichesky, (2006) las escuelas que trabajan en contextos de pobreza, con alumnos y alumnas (niños, adolescentes, jóvenes y/o adultos) con escasas oportunidades sociales, deben enfrentar una serie de problemáticas (socioeconómicas, pedagógicas, institucionales) para desarrollar una enseñanza de calidad. Una de sus consecuencias - aún sin resolver en la Argentina y en los países de la región- es la cristalización de trayectorias educativas 
signadas por la discontinuidad, escasos aprendizajes y, en casos más graves, abandono educativo. Esta situación socioeducativa profundiza y amplía las brechas de desigualdad social.

Cuando se interroga a los jóvenes de la región (América Latina y el Caribe; datos de la CEPAL, 2003) respecto de los motivos de la deserción escolar, mencionan principalmente los de índole económica, como la búsqueda de empleo y carencias, además de los familiares, como embarazo, maternidad o responsabilidad en los quehaceres del hogar, que evidentemente afectan de manera preferencial a las mujeres. Al mismo tiempo, entre un $10 \%$ y un $25 \%$ de los y las jóvenes señalan la falta de interés y el mal desempeño escolar. Y son justamente sobre estos últimos factores donde una formación docente de calidad puede incidir de manera decisiva para acrecentar la retención y revertir las cifras de exclusión educativa. Todo esto, sin abandonar el objetivo de producir aprendizajes de calidad.

\section{Un modelo de formación centrado en los contextos de desempeño}

Consideramos que se torna imperioso un desarrollo profesional docente estrechamente ligado a la escuela y orientado a integrar las perspectivas teóricas y prácticas a dicho proceso; acciones que involucran la participación de los y las docentes en los proyectos de planificación e implementación. Este modelo de desarrollo profesional, cada vez más preponderante en muchos países, muestra que el apoyo que se brinda al docente necesita centrarse en el contexto de su desempeño. Supone acciones pensadas para acortar la distancia entre las pro- puestas curriculares y la realidad de las aulas. En nuestro caso, de aulas con jóvenes expuestos a situaciones de vulnerabilidad extrema, jóvenes en situación de libertad asistida, madres y padres adolescentes, que viven en condiciones de gran precariedad, sin servicios públicos, con inseguridad extrema, rodeados de violencia y violentamente, en medio de conductas destructivas, con la discriminación en el tratamiento personal y de sus espacios, en aislamiento, en soledad, en la invisibilidad de la sociedad.

En el trabajo que proponemos, y que desde diferentes proyectos venimos abordando, intentamos hallar orientaciones respecto de qué debe tener en cuenta una formación de formadores para que los futuros profesores puedan luego colaborar con la retención escolar y el desarrollo integral de estos jóvenes alumnos. Compartimos la idea de que la medida verdadera del éxito de un programa de desarrollo profesional de los profesores, es el aumento significativo en el aprendizaje de los estudiantes (AbuSharbain, 2002).

Nos preguntamos por cómo favorecer un desarrollo profesional docente que promueva una educación científica de calidad en los jóvenes de los sectores más vulnerables socialmente. En este sentido, coincidimos con Sánchez (2001) en considerar que el desarrollo profesional docente es un proceso continuo sustentado en las necesidades prácticas, que implica la participación y la reflexión sobre el desempeño, y que constituye un proceso de construcción mediante el cual los docentes desarrollan estrategias cognitivas que les permiten valorar su trabajo profesional. 
Pensar que la formación que se brinda al docente debe centrarse en el contexto de su desempeño, supone acciones pensadas para apoyar a los docentes y para acortar la distancia entre las propuestas curriculares y la realidad de las aulas (Perrenoud, 2004). Se trata de producir una revaloración de la escuela como ámbito donde se aprende, de allí que bajo esta perspectiva, se ha generado un énfasis en las investigaciones sobre el estudio local y en contexto, sobre la experiencia vivida; la experiencia se convierte, así, en fuente de apropiación de saberes.

\section{Retención y calidad}

El objetivo del trabajo que venimos realizando en la formación del profesorado de biología en la Universidad de Buenos Aires (Argentina), es contribuir al desarrollo profesional docente mediante la producción de prácticas reflexivas que orienten la intervención didáctica, con el fin de mejorar la retención y la calidad de los aprendizajes científicos en escuelas que atienden jóvenes en situación de vulnerabilidad extrema.

Coincidimos con Machado (2002) en que mejorar la calidad de la educación en las escuelas significa promover procesos pedagógicos en el aula. Esto requiere establecer cambios profundos en todas las dimensiones, principalmente en la formación de los docentes y en como éstos plantean el desarrollo de su labor profesional en las instituciones educativas.

Nuestra hipótesis de trabajo es que si se espera lograr la modificación y adecuación de modelos teóricos y prácticas docentes tradicionales, para dar respuesta a las demandas crecientes que plantean los contextos educativos más desfavorecidos, la formación profesional docente deberá organizarse alrededor de una práctica reflexiva que promueva en los educadores y educadoras el desarrollo de estrategias y dispositivos que les permitan abordar los desafíos que implica la retención educativa y la inclusión de los jóvenes más vulnerables.

La práctica reflexiva lleva a reconocer el carácter arbitrario del modelo -didáctico- que se presenta como natural. En tanto no se visualice como producción social, no podrá cuestionarse, explicarse ni modificarse (Alliaud, 1993). Al mismo tiempo, esta práctica reflexiva debe sustentarse en el conocimiento profundo de los contextos en los cuales opera; como menciona Pennac (2008), la reflexión tiene que darse necesariamente en la acción misma de enseñar y no por fuera, como una ciencia abstracta.

\section{Qué pretendemos con la formación inicial docente}

Sostenemos que una formación docente inicial debe potenciar no sólo aquellos elementos más estrechamente ligados al desarrollo profesional docente, sino también a la escuela y a los y las jóvenes estudiantes, en relación con el desarrollo profesional, fomentamos:

- La articulación entre la investigación didáctica, la realización de observaciones reflexivas de clases y la planificación de prácticas innovadoras de enseñanza, atendiendo a contextos y necesidades reales de los estudiantes de las escuelas involucradas en el proyecto.

- El fortalecimiento de las habilidades comunicativas, docentes, solidarias y afectivas del futuro profesor.

- La interacción dialógica con colegas de más experiencia y el desarrollo de estrategias colaborativas. 
- La incorporación de nuevas variables de las prácticas educativas y el desarrollo de una actitud reflexiva sobre ellas.

- La toma de conciencia de la importancia del rol del docente como agente de cambio social.

- El inicio en la formulación y puesta en el aula de estrategias de innovación educativa.

- La capacitación de los futuros profesores para trabajar en contextos escolares diversos y, sobre todo, para trabajar en la prevención de la deserción escolar, en escuelas que atienden jóvenes en situación de vulnerabilidad social extrema.

En relación con las escuelas, procuramos:

- El fortalecimiento de vínculos de trabajo permanente entre la escuela y la formación docente.

- El apoyo a la tarea del docente en servicio.

- El posicionamiento de la escuela como ámbito que contribuye a la formación real de los docentes, ya que permite el conocimiento del contexto social, cultural e institucional en el cual se deberá desplegar el accionar docente, y para el que la formación deberá estar dirigida.

- El reconocimiento de la escuela como espacio de construcción de saber didáctico.

En relación con los estudiantes de nivel medio, perseguimos:

- El incremento del apoyo emocional, social e intelectual de los jóvenes.

- La construcción de mejores aprendizajes.

- El aumento de la retención escolar.

Es deseable que la formación docente sea concebida como un proceso continuo, flexible y contextualizado, que se apoye en la permanente reflexión, estimulando el valor de lo axiológico y considerando los juicios críticos sobre los valores sociales, éticos y políticos (Valeiras y Meinardi, 2007), es importante planificar diferentes alternativas o modalidades que se ajusten a las necesidades y posibilidades del profesorado como a las exigencias de su contexto de actuación profesional. En este sentido, el trabajo de los grupos de innovación se sustenta en la combinación del trabajo individual y grupal que permite, a través del debate, la reflexión conjunta acerca del desempeño profesional con la puesta en común de las estrategias de innovación aplicadas (Tirados y Maura, 2007).

La indispensable implicación de los profesores en su proceso formativo se inicia con la participación de éstos en el estudio de dichas necesidades. Esto hace que todo programa de formación deba estar precedido de un análisis de necesidades formativas para que cumpla con una de las características más importantes de dicha acción: la pertinencia, ya que un programa es pertinente si responde a las necesidades existentes. Como señalan diversos autores, tener en cuenta las necesidades del profesorado es un óptimo inicio para la mejora de la calidad de la enseñanza (Cox, 1994).

\section{Conclusiones}

Consideramos que las acciones formadoras deben llevar a que el profesorado pueda producir una fundamentación teórica de su actuación, congruente con los nuevos conocimientos que se van elaborando sobre los procesos de enseñanza y de aprendizaje de las ciencias, y a un conocimiento para vincular estos conocimientos con su práctica. Como también es necesario que se den cambios en el sistema de valores y actitudes, es 
imprescindible que todo el proceso de formación se vincule con una reflexión crítica y que contemple el contexto sociocultural de actuación y el mundo emocional del que enseña.

Nuestra propuesta es incidir sobre la formación de los docentes, provocando la reflexión in situ sobre las prácticas de enseñanza y su reconstrucción, destacando la importancia de articular la formación de los docentes, tanto inicial como en servicio con la investigación, para producir nuevas y mejores prácticas para la enseñanza de las ciencias.

Para lograrlo, desarrollamos un conjunto de programas y acciones variadas

\section{Bibliografía}

AA.VV. (2009). Superar la desigualdad: por qué es importante la gobernanza. Informe de Seguimiento de la Educación en el Mundo 2009. UNESCO.

AbuSharbain, E. (2002). Enhancing Inservice Teacher's Constructivist Epistemology through the Development and Redesign of Inquiry-based Investigations together with their Students. Electronic Journal of Science Education, 7 (1).

Alliaud, A. (1993). Los maestros y su historia. Buenos Aires: Centro Editor de América Latina.

CEPAL. (2003). Panorama Social de América Latina 2002-2003. Santiago de Chile: Publicación de Naciones Unidas.

Borzese, D. y Bottinelli, L. (2005). Inclusión con calidad educativa para todos los jóvenes. Algunos datos para la reflexión y la acción. En Krichesky, M. (Comp.). Adolescencia e Inclusión educativa: Un derecho en cuestión. México: Novedades Educativas. que involucran a los estudiantes, futuros profesores, en actividades que los llevan a participar de diferentes maneras en los procesos escolares, a permanecer y colaborar con las escuelas, los docentes y los alumnos a lo largo de un año de trabajo.

Al mismo tiempo, como formamos parte de un equipo que investiga en la formación docente, intentamos construir una didáctica que pueda dar respuesta a las prácticas de enseñanza que se requieren en las escuelas, sin olvidar que todo proceso de formación docente debe servir también para la formación de nosotros mismos, los formadores de formadores.

Cox, B. (1994). Practical Pointers for University Teachers. London: Kogan Page.

Croce, A. (2005). De las resistencias internas para incluir a los adolescentes y jóvenes en el sistema educativo. En Krichesky, M. (Comp.). Adolescencia e Inclusión educativa: Un derecho en cuestión. México: Novedades Educativas.

Duro, E. (2005). Adolescencia y políticas públicas. De la invisibilidad a la necesaria centralidad. En Krichesky, M. (Comp.). Adolescencia e Inclusión Educativa: Un derecho en cuestión. México: Novedades Educativas.

Feldman, D. (2005). Currículo e Inclusión educativa. En Krichesky, M. (comp.). Adolescencia e Inclusión educativa: Un derecho en cuestión. México: Novedades Educativas.

Fundación SES (2007). Sustentabilidad, Educación y Solidaridad. Obtenido desde www.fundses.org.ar 
Gentili, P. (1998). A falsificação do consenso. Simulacro e imposição na reforma educacional do neoliberalismo. Petrópolis: Vozes.

Gentili, P. (2007). Desencanto y utopía. La educación en el laberinto de los nuevos tiempos. Rosario: Homo Sapiens.

Gentili, P. (2009). Marchas y contramarchas. El derecho a la educación y las dinámicas de exclusión incluyente en América Latina (a sesenta años de la Declaración Universal de los Derechos Humanos). Revista Iberoamericana de Educación, 49, 19-57.

Gentili y Alencar, C. (2001). Educar na esperança em tempos de desencanto. Petrópolis: Vozes.

INDEC. Instituto Nacional de Estadística y Censos, Argentina. Censo Nacional 2001, Obtenido en marzo 2009, desde http://www.indec.mecon.ar

Jacinto, C. y Terigi, F. (2007). ¿Qué hacer ante las desigualdades en la educación secundaria? Aportes a la experiencia latinoamericana. Buenos Aires: IIPEUNESCO.

Krichesky, M. (Coord.) (2006). Escuela y comunidad: desafíos para la inclusión educativa. Buenos Aires: Ministerio de Educación, Ciencia y Tecnología de la Nación.

Machado, A. L. (2002). Formación docente: un aporte a la discusión. Chile: Unesco.

Meinardi, E. (2007). Reflexiones sobre la formación inicial de los profesores de Biología. Revista de Educación en Biología. 10 (2), 48-54.
Oficina regional de Educación para América Latina y el Caribe. (2003). Alcanzando las metas educativas. Santiago de Chile: UNESCO.

Pennac, D. (2008). Mal de Escuela, Barcelona: Mondadori.

Perrenoud, P. (2004). Desarrollar la práctica reflexiva en el oficio de enseñar. Barcelona: Graó.

Sánchez, J. A. (2001). El desarrollo profesional del docente universitario. Revista Universidades. 22.

Sistema de Información de Tendencias Educativas en América Latina. (2008). Resumen Estadístico. Totales regionales. Obtenido desde http://www. siteal.iipe-oei.org/

Tirados, R. M. y Maura, V. (2007). Diagnóstico de necesidades y estrategias de formación docente en las universidades. Revista Iberoamericana de Educación, 43:6.

UNESCO (2008). Educación: barreras en el camino El Correo de la UNESCO. Autor (es): Comunicado de prensa No. 2008-119. Fuente: UNESCOPRENSA. 25-11-2008.

Valeiras, N. y Meinardi, E. (2007). La enseñanza de la biología, las reformas educativas y la realidad del profesorado en Argentina. Alambique, 51, 58-65. 